\title{
A More Accurate Reconstruction Method for Detecting Large-depth Defects in Plates Using SH Guided Waves
}

\author{
Yihui Da ${ }^{\mathrm{a}}$, Qi Li ${ }^{\mathrm{a}}$, Bin Wang ${ }^{\mathrm{a}}$, Dianzi Liu ${ }^{\mathrm{b}, *}$, Zhenghua Qian ${ }^{\mathrm{a}, *}$ \\ a State Key Laboratory of Mechanics and Control of Mechanical Structures, College of Aerospace \\ Engineering, Nanjing University of Aeronautics and Astronautics, Nanjing 210016, China \\ ${ }^{\mathrm{b}}$ School of Engineering, University of East Anglia, Norwich NR4 7TJ, UK \\ *Corresponding authors: qianzh@nuaa.edu.cn and dianzi.liu@uea.ac.uk
}

\begin{abstract}
The inspection of thickness thinning defects and corrosion defects is greatly significant for the health prediction of plate structures. The main aim of this research is to propose a novel and effective approach to achieve the accurate and rapid detection of arbitrary defects using shear horizontal (SH) guided waves, particularly for large-depth and complex defects. The proposed approach combines the quantitative detection of Fourier transform (QDFT) with a reference model-based strategy to improve the accuracy of large-depth defect detection. Since the shallow defect profile is theoretically constructed by inverse Fourier transform of the product of reflection coefficients and integral coefficients of reference models, the unknown large-depth defect can be initially assessed using the relevant information from a predefined reference model. By iteratively updating the integral coefficients of reference models, the accuracy of reconstruction of large-depth defects is much improved. To achieve the converged defect profile, a termination criterion, the root mean square error (RMSE), is applied to guarantee the construction of defects with a high level of accuracy. Moreover, the hybrid finite element method (HFEM) is used to simulate the propagation of SH guided waves in plates for calculating the reflection coefficients of plates with defects. Finally, to demonstrate the capability of the developed reconstruction method for defect detection in terms of accuracy and efficiency, three types of large-depth defect profiles, i.e., a rectangular flaw, a double-rectangular flaw, and a complex flaw, are examined. Results show that the discrepancy between the predicted defect profile and the real one is quite small, even in the largest-depth defect case where the defect depth is equal to 0.733 times the plate thickness, the minimal difference is observed. It is noted that the fast convergence of the proposed approach can be achieved by no more than ten updates for the worst case.
\end{abstract}

KEYWORDS Large-depth defect, SH guided wave, Iterative strategy, Reference model, Hybrid FEM 


\section{Introduction}

Detecting surface corrosion defects in plate structures is a meaningful topic. Accurately quantifying the thickness loss and greatly enhancing the efficiency of detection are of growing importance. Traditional ultrasonic detection methods always involve some assumptions, such as the Born approximation (BA), to approximately evaluate the wave fields in the defect areas or near the defect boundaries. Using the lowfrequency guided wave diffraction tomography [1] to detect defects, finite element simulations of a plate were performed for the accurate thinning reconstruction. Based on the Born approximation and the far-field approximation, the depth of plate thinning can be expressed as a function of the horizontal coordinate by performing the inverse Fourier transform of the reflection coefficients at various frequencies [2]. Furthermore, the reflection full-waveform inversion (FWI) has been recently utilized to improve the accuracy of imaging the Earth interior by separating the tomographic gradient from the reflectivity gradient using the Born approximation during forward modeling [3]. However, it is certain that the Born approximation is more valid for weakly scattered sources rather than defects of arbitrary sizes mentioned in $[4,5]$.

In order to improve the quality of defect reconstruction and enhance the universality of detection methods, various improved approaches have been proposed. A modified extended Born approximation (MEBA) was presented for efficient three-dimensional simulations and inversion of geophysical frequencydomain electromagnetic (EM) data caused by a targeted object lodged in a layered half-space [6]. A new decomposition of the forward scattering map was derived to reveal a previously unknown approximate bilinear forward scattering relation. Results indicated that the new linear inverse scattering approach could be more broadly applicable than the classical Born-approximation-based imaging [7]. A novel method based on the matrix completion (MC) paradigm was exploited to image the weak and sparse scatters in heavy noise conditions [8]. A modified Born iterative method (BIM) by which magnetic fields were analyzed and processed was investigated to retrieve more accurate images within the medical emergency time frame [9]. The Bayesian approximation error approach was used to partially recover the error structure induced by the Born approximation [10]. A new non-linear Born iterative reconstruction method with USguided depth-dependent $\ell 1$ sparse regularization was proposed to improve diffuse optical tomography (DOT) reconstruction by incorporating a priori lesion depth and shape information from the co-registered US image [11]. To obtain the non-linear tomographic radar imaging, Sorsa et al. [12] introduced and numerically evaluated a multigrid solver, which enabled the fast and robust inversion of sparse time- 
domain data with a mathematical full-wave approach utilizing a higher-order Born approximation. Summarily, the usage of the modified Born approximation and aforementioned assumptions can improve the imaging precision and efficiency.

Experimentally, guided waves have been widely explored as a promising inspection tool for nondestructive evaluation [13-17]. From the computational point of view, the hybrid finite element method (FEM), as a fast modeling instrument of guided waves scattering, were introduced in [18, 19]. This method was also adopted to simulate ultrasonically generated waves propagating in an infinitely long pipe that included a notch [20]. Then, a combined analytical finite element model approach (CAFA) was proposed for the accurate, efficient, and versatile simulation of 2D Lamb wave propagation and interaction with damage [21]. For the inverse algorithm, the iterative technique, as an effective means that ensures the reliability of detection results, has been applied to the acoustic tomography [22-27]. In the thickness maps of guided wave tomography, the iterative hybrid algorithm for robust breast ultrasound tomography (HARBUT) was used to improve the accuracy of reconstructions of defects [28]. An iterative S-wave velocity inversion method guided by image registration was developed by Yang [29]. A guided wave tomography method based on full waveform inversion (FWI) was considered to discretize the frequency components from low to high frequencies [30].

In this paper, a more accurate reconstruction method, which integrates the numerical modeling of forward guided wave problems using the hybrid FEM with the algorithm for defect reconstruction, has been proposed to improve the efficiency of defect detection and increase the accuracy of imaging. For forward problems, the developed hybrid FEM technique in Section 2.1 has been applied to obtain the reflected coefficients of shear horizontal $(\mathrm{SH})$ waves induced by the same mode of incident waves. For inverse problems, the quantitative detection of Fourier transform (QDFT) approach has been briefly introduced as an efficient inspection method for 2D structures in Section 2.2. To address the reconstruction of large-depth defects, a modified QDFT method, called the QDFTU, has been proposed in Section 3. Finally, numerical experiments using QDFTU have been examined and discussions on reconstruction results have been presented in Section 4. Following that, the conclusion has been drawn in Section 5.

\section{Numerical Modeling and Algorithm for Defect Reconstruction}

\subsection{Numerical Modeling of Scattered Waves Using Hybrid FEM}


The hybrid FEM (HFEM) is adopted to develop the numerical model for the calculations of scattered fields in plates, where the finite element analysis is only performed in the region surrounding the defect (see Fig. 1). Both the incident and reflected waves propagate through $S_{2}$, whilst the transmitted waves travel through $S_{1}$. Nodal displacements and forces on the two cross-sections, $S_{1}$ and $S_{2}$, consist of infinite components induced by different wave modes with unknown amplitudes, which can be formulated by the semi-analytical finite element $[32,33]$. Obviously, most of the non-propagating modes will quickly die off, and only the propagating and a few non-propagating modes will be used to calculate the scattered fields.

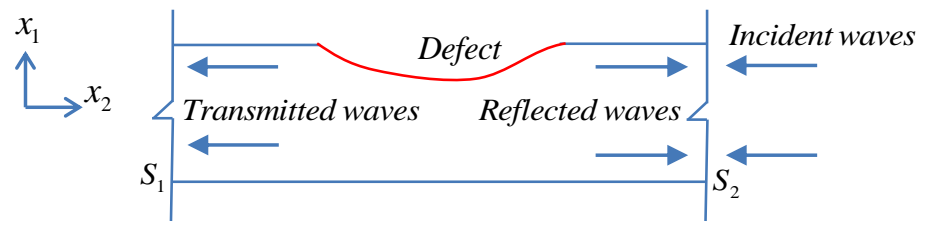

Fig. 1. Scattered fields in plates are calculated by the hybrid FEM and the incident SH waves propagating along the minus direction of the $x_{2}$-axis. The red line represents the defect boundary, and the blue line means the non-defective region. $S_{1}$ and $S_{2}$ denote the cross sections on which the incident waves transmit and reflect, respectively.

According to the conventional FEM, the matrix equation of motion is written as:

$\delta\left([\boldsymbol{q}]^{\mathrm{H}}\right) \boldsymbol{S} \boldsymbol{q}=\delta\left([\boldsymbol{q}]^{\mathrm{H}}\right) \boldsymbol{P}$

where

$\boldsymbol{S}=\boldsymbol{K}-\omega^{2} \boldsymbol{M}$

$\boldsymbol{K}$ and $\boldsymbol{M}$ are the global stiffness and mass matrices, $\omega$ represents the circular frequency, and $\boldsymbol{q}$ and $\boldsymbol{P}$ are nodal displacements and force vectors, respectively. The superscript ' $H$ ' means conjugate transpose, and ' $\delta$ ' denotes the first variation.

$\delta\left(\left[\begin{array}{c}\boldsymbol{q}_{\mathrm{I}} \\ \boldsymbol{q}_{\mathrm{B}}^{\mathrm{H}}\end{array}\right]^{\mathrm{H}}\right)\left[\begin{array}{ll}\boldsymbol{S}_{\mathrm{II}} & \boldsymbol{S}_{\mathrm{IB}} \\ \boldsymbol{S}_{\mathrm{BI}} & \boldsymbol{S}_{\mathrm{BB}}\end{array}\right]\left[\begin{array}{c}\boldsymbol{q}_{\mathrm{I}} \\ \boldsymbol{q}_{\mathrm{B}}\end{array}\right]=\delta\left(\left[\begin{array}{c}\boldsymbol{q}_{\mathrm{I}} \\ \boldsymbol{q}_{\mathrm{B}}\end{array}\right]^{\mathrm{H}}\right)\left[\begin{array}{l}\boldsymbol{P}_{\mathrm{I}} \\ \boldsymbol{P}_{\mathrm{B}}\end{array}\right]$

where $\boldsymbol{P}_{\mathrm{I}}=\mathbf{0}$. The nodal displacement vector $\boldsymbol{q}_{\mathrm{B}}=\left\{\begin{array}{l}\boldsymbol{q}_{S_{1}} \\ \boldsymbol{q}_{S_{2}}\end{array}\right\}$ and force vector $\boldsymbol{P}_{\mathrm{B}}=\left\{\begin{array}{l}\boldsymbol{P}_{S_{1}} \\ \boldsymbol{P}_{S_{2}}\end{array}\right\}$ at cross sections $S_{1}$ and $S_{2}$ contain incident, reflected and transmitted waves as below:

$\boldsymbol{q}_{S_{1}}=\boldsymbol{q}_{S_{1}}^{\mathrm{inc}}+\boldsymbol{q}_{S_{1}}^{\mathrm{tra}}$

$\boldsymbol{q}_{S_{2}}=\boldsymbol{q}_{S_{2}}^{\mathrm{inc}}+\boldsymbol{q}_{S_{2}}^{\mathrm{ref}}$

and 
$\boldsymbol{P}_{S_{1}}=\boldsymbol{P}_{S_{1}}^{\mathrm{inc}}+\boldsymbol{P}_{S_{1}}^{\mathrm{tra}}$

$\boldsymbol{P}_{S_{2}}=\boldsymbol{P}_{S_{2}}^{\mathrm{inc}}+\boldsymbol{P}_{S_{2}}^{\mathrm{ref}}$

where the superscripts 'inc', 'tra' and 'ref' indicate the incident, transmitted and reflected waves, respectively. They can be expressed by the modal expansion of the wave functions of the undamaged waveguide [32].

The matrix equation is simplified as

$G\left[\begin{array}{l}q_{\mathrm{I}} \\ \widetilde{A}\end{array}\right]=T$

where $\left.\left.\boldsymbol{G}=\left\{\left[\begin{array}{cc}\boldsymbol{I} & \mathbf{0} \\ \mathbf{0} & {[\widetilde{\boldsymbol{\Phi}}}\end{array}\right]^{\mathrm{H}}\right]\left[\begin{array}{cc}\boldsymbol{S}_{\mathrm{II}} & \boldsymbol{S}_{I B} \\ \boldsymbol{S}_{\mathrm{BI}} & \boldsymbol{S}_{\mathrm{BB}}\end{array}\right]\left[\begin{array}{cc}\mathbf{I} & \mathbf{0} \\ \mathbf{0} & \widetilde{\boldsymbol{\Phi}}\end{array}\right]-\left[\begin{array}{cc}\mathbf{I} & \mathbf{0} \\ \mathbf{0} & {[\widetilde{\boldsymbol{\Phi}}}\end{array}\right]^{\mathrm{H}}\right]\left[\begin{array}{cc}\mathbf{0} & \mathbf{0} \\ \mathbf{0} & \tilde{\boldsymbol{t}}\end{array}\right]\right\}$

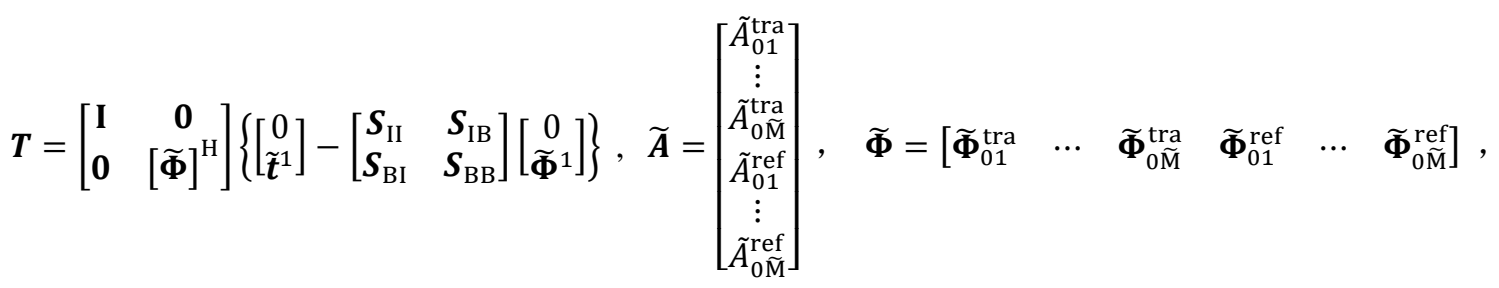

$\widetilde{\boldsymbol{\Phi}}^{1}=\left[\begin{array}{l}\widetilde{\boldsymbol{\Phi}}_{0 m}^{1} \\ \widetilde{\boldsymbol{\Phi}}_{0 m}^{2}\end{array}\right], \quad \tilde{\boldsymbol{t}}^{1}=\left[\begin{array}{l}\boldsymbol{t}_{0 m}^{1} \\ \boldsymbol{t}_{0 m}^{2}\end{array}\right], \quad \tilde{\boldsymbol{t}}=\left[\begin{array}{llllll}\tilde{\boldsymbol{t}}_{01}^{\mathrm{tra}} & \cdots & \tilde{\boldsymbol{t}}_{0 \bar{M}}^{\text {tra }} & \tilde{\boldsymbol{t}}_{01}^{\text {ref }} & \cdots & \tilde{\boldsymbol{t}}_{0 \widetilde{M}}^{\text {ref }}\end{array}\right], \quad \boldsymbol{I}$ is an identity matrix, the subscripts I and B mean interior nodes and boundary nodes, respectively, $\boldsymbol{q}_{\mathrm{I}}$ is the displacement vector of the interior nodes, $\widetilde{M}$ is the total number of non-propagating waves and guided waves corresponding to different frequencies, $\widetilde{\boldsymbol{A}}$ is the modified coefficients for scattered fields. $\widetilde{\boldsymbol{\Phi}}$ and $\widetilde{\boldsymbol{t}}$, respectively, denote the nodal displacements and forces induced by a unity amplitude of reflected and transmitted waves propagating through the cross sections $S_{1}$ and $S_{2}$. And $\widetilde{\boldsymbol{\Phi}}^{1}$ and $\tilde{\boldsymbol{t}}^{1}$, respectively, represent the nodal displacements and forces inducted by incident waves through the cross sections. For more details about the calculation of scattered fields using HFEM, refer to our previous paper [31].

\subsection{A Brief Review of QDFT for Reconstruction of Defects}

According to the reciprocal theory [34], the simple relationship between the reflected displacement at the monitoring point caused by the defect profile and the total field at the defect boundary can be established as:

$$
\int_{S}\left[u_{i}^{\mathrm{total}}(\boldsymbol{x}) \tilde{T}_{i j}^{\alpha}(\boldsymbol{x}-\boldsymbol{X})\right] \boldsymbol{n}_{j} \mathrm{~d} S(\boldsymbol{x})=u_{\alpha}^{\mathrm{ref}}(\boldsymbol{X})(\boldsymbol{X} \notin V ; i, j, \alpha=1,2)
$$


where the field and source point (or monitoring point) are defined by the coordinates $\left(x_{1}, x_{2}\right)$ and $\left(X_{1}, X_{2}\right)$, respectively. The superscripts 'total' and 'ref' represent the total field and reflected field, respectively. $\tilde{T}_{i j}^{\alpha}(\boldsymbol{x}-\boldsymbol{X})$ indicates the stress field calculated by Green's function of the current structure, where $\alpha$ is the direction of a unit load at the source point. $u_{i}^{\text {total }}(\boldsymbol{x})$ represents the displacement vector, $\boldsymbol{n}_{j}$ denotes the normal vector of the defect boundary $S(\boldsymbol{x})$, and ' $V$ ' depicts the defect area. It is noted that the source point $\boldsymbol{X}$ locates in the reflected region, and the traction-free boundary condition is omitted.

To facilitate the theoretical analysis, the separation of variables is utilized as follows:

$\tilde{T}_{i j}^{\alpha}(\boldsymbol{x}-\boldsymbol{X})=\tilde{P}_{i j}^{\alpha}\left(x_{1}-X_{1}\right) \mathrm{e}^{-\mathrm{i} \xi\left(x_{2}-X_{2}\right)} ; u_{\alpha}^{\mathrm{ref}}(\boldsymbol{X})=A_{\alpha}^{\mathrm{ref}}\left(X_{1}\right) \mathrm{e}^{\mathrm{i} \xi X_{2}} ; u_{i}^{\text {total }}(\boldsymbol{x}) \approx A_{i}^{\mathrm{inc}}\left(x_{1}\right) \mathrm{e}^{-\mathrm{i} \xi x_{2}}$

where $\xi$ is the wavenumber, i denotes $\sqrt{-1}, \tilde{P}_{i j}^{\alpha}, A_{i}^{\text {inc }}\left(x_{1}\right)$ and $A_{\alpha}^{\text {ref }}$ represent amplitudes of waves. $\mathrm{e}^{-\mathrm{i} \xi x_{2}}$, $\mathrm{e}^{-\mathrm{i} \xi\left(x_{2}-X_{2}\right)}$ and $\mathrm{e}^{\mathrm{i} \xi X_{2}}$ are terms of wave propagation. And the total field $u_{i}^{\text {total }}(\boldsymbol{x})$ is approximately replaced by the incident field due to the assumption of Born approximation. In this paper, the time harmonic term is omitted.

Applying Gauss's divergence theorem, Eq. (2) can be further rewritten as:

$\int_{-\infty}^{+\infty} \mathrm{e}^{-2 \mathrm{i} \xi x_{2}} \mathrm{e}^{\mathrm{i} \xi X_{2}} \int_{h-\eta\left(x_{2}\right)}^{h}\left\{\left[A_{i}^{\text {inc }}\left(x_{1}\right) \tilde{P}_{i 1}^{\alpha}\left(x_{1}-X_{1}\right)\right]_{, 1}+Q\right\} \mathrm{d} x_{1} \mathrm{~d} x_{2} \approx A_{\alpha}^{\mathrm{ref}}\left(X_{1}\right) \mathrm{e}^{\mathrm{i} \xi X_{2}}$

$Q=-2 \mathrm{i} \xi A_{i}^{\mathrm{inc}}\left(x_{1}\right) \tilde{P}_{i 2}^{\alpha}\left(x_{1}-X_{1}\right)$

where the subscript ', 1 ' represents $\frac{\partial}{\partial x_{1}}, h$ and $\eta\left(x_{2}\right)$ signify the plate thickness and the defect depth, respectively.

Furthermore, using the modes of the incident guided wave, the reflected waves can be formulated as

$u_{\alpha}^{\mathrm{ref}}(\boldsymbol{X})=A_{\alpha}^{\mathrm{ref}}\left(X_{1}\right) \mathrm{e}^{\mathrm{i} \xi X_{2}}=C^{\mathrm{ref}}(\xi) A_{\alpha}^{\mathrm{inc}}\left(X_{1}\right) \mathrm{e}^{\mathrm{i} \xi X_{2}}$

where the reflection coefficients are given by $C^{\mathrm{ref}}(\xi)=\frac{A_{\alpha}^{\mathrm{ref}}\left(X_{1}\right)}{A_{\alpha}^{\mathrm{inc}}\left(X_{1}\right)}$. 
In the equations above, the source point is far from the defect region $V$, and the integrand $\left[A_{i}^{\text {total }}\left(x_{1}\right) \tilde{P}_{i 1}^{\alpha}\left(x_{1}-X_{1}\right)\right]_{, 1}$ and $Q$ have no singularity in the interval of integration. Therefore, there exists the original function as follows:

$\left.\widetilde{W}\left(x_{1}, \xi\right)\right|_{h-\eta\left(x_{2}\right)} ^{h}=\int_{h-\eta\left(x_{2}\right)}^{h}\left\{\left[A_{i}^{\text {total }}\left(x_{1}\right) \tilde{P}_{i 1}^{\alpha}\left(x_{1}-X_{1}\right)\right]_{, 1}+Q\right\} \mathrm{d} x_{1}$

Substituting Eqs. (3), (5) and (6) into Eq. (4), one has:

$$
\begin{aligned}
\int_{-\infty}^{+\infty} \mathrm{e}^{-2 \mathrm{i} \xi x_{2}} \mathrm{e}^{\mathrm{i} \xi x_{2}} & \eta\left(x_{2}\right) \lim _{\eta\left(x_{2}\right) \rightarrow 0} \frac{\widetilde{W}(h, \xi)-\widetilde{W}\left(h-\eta\left(x_{2}\right), \xi\right)}{h-\left(h-\eta\left(x_{2}\right)\right)} \mathrm{d} x_{2} \\
& \left.\approx \int_{-\infty}^{+\infty} \mathrm{e}^{-2 \mathrm{i} \xi x_{2}} \mathrm{e}^{\mathrm{i} \xi x_{2}} \eta\left(x_{2}\right) \frac{\mathrm{d} \widetilde{W}\left(x_{1}, \xi\right)}{\mathrm{d} x_{1}}\right|_{x_{1}=h} \mathrm{~d} x_{2} \approx C^{\mathrm{ref}}(\xi) A_{\alpha}^{\mathrm{inc}}\left(X_{1}\right) \mathrm{e}^{\mathrm{i} \xi x_{2}}
\end{aligned}
$$

Replacing $R(k)$ with $\left.\frac{\mathrm{d} \widetilde{W}\left(x_{1}, \xi\right)}{\mathrm{d} x_{1}}\right|_{x_{1}=h}$ in Eq. (7), the defect function is derived using the inverse Fourier transform, and one has

$\eta\left(x_{2}\right) \approx \frac{1}{2 \pi} \int_{-\infty}^{+\infty} C^{\mathrm{ref}}(k) B(k) \mathrm{e}^{\mathrm{i} k x_{2}} \mathrm{~d} k$

where $B(k)=\frac{A_{\alpha}^{\mathrm{inc}}\left(X_{1}\right)}{R(k)}$ and $k=2 \xi$.

\section{The Modified QDFT for Reconstruction of Large-depth Defects}

According to the QDFT described in Section 2, Eq. (8) illuminates that the function $B(k)$ only depends on the wavenumber $k$ when defects can be considered as weak scattering sources. For the plate with largedepth defects, a predefined reference model can be chosen to initially approximate $B(k)$ of the inspected plate using $B_{0}(k)$ obtained from the given reference model. Therefore, Eq. (8) can be rewritten as

$\eta_{1}\left(x_{2}\right) \approx \frac{1}{2 \pi} \int_{-\infty}^{+\infty} C^{\mathrm{ref}}(k) B_{0}(k) \mathrm{e}^{\mathrm{i} k x_{2}} \mathrm{~d} k$

where the $C^{\mathrm{ref}}(k)$ is obtained by simulations or experiments for the specimen plate.

It is noted that there always exists the discrepancy between the predicted result by the QDFT and a real defect, because the defect in the reference model cannot be selected exactly the same as the unknown flaw in the specimen plate. Therefore, it is extremely difficult to achieve the accuracy of defect reconstruction 
merely by a single simulation of QDFT, especially for the case of large-depth defects. To address this issue, an improved QDFT with the updating strategy for $B_{i}(k)$, called the QDFTU, is proposed in this section. The QDFTU has the ability to predict the large-depth defect profile with higher levels of accuracy and efficiency throughout iterative calculations of $B_{i}(k)$ obtained from the reference model. The update of $B_{i}(k)$ will terminate until a convergence criterion for two consecutive reconstructions is satisfied. The developed approach works towards the converged large-depth defect profile and guarantees the reliability of the reconstruction result. Obviously, the remarkable advantage of QDFTU has been demonstrated by avoiding the derivation of closed-form Green's functions and facilitating the detection of complex largedepth defects. This leads to quantitative defect detection with a high level of accuracy.

The details of the QDFTU approach include five steps as follows:

Step 1: To choose a pre-defined reference model with the defect profile of $\eta_{0}\left(x_{1}\right)$;

Step 2: To calculate the reflection coefficients $C_{0}^{\text {ref }}(k)$ of the reference model in Step 1 by HFEM, and obtain $B_{0}(k)$ by inverse operation of Eq. (9), i. e., $B_{0}(k) \approx\left[\int_{-\infty}^{+\infty} \eta_{0}\left(x_{2}\right) \mathrm{e}^{-\mathrm{i} k x_{2}} \mathrm{~d} x_{2}\right] / C_{0}^{\text {ref }}(k)$;

Step 3: To reconstruct the defect profile $\eta_{1}\left(x_{1}\right)$ using Eq. (9);

Step 4: To judge whether the difference between $\eta_{1}\left(x_{1}\right)$ and $\eta_{0}\left(x_{1}\right)$ satisfies the convergence condition. If the result meets the condition, the final result is achieved; otherwise, to update $\eta_{0}\left(x_{1}\right)$ of the reference model with $\eta_{1}\left(x_{1}\right)$;

Step 5: To repeat Steps 2 to 4 until the convergence condition is satisfied.

The flowchart for the QDFTU approach has been given in Fig. 2. The reflection coefficients $C_{0}^{\text {ref }}(k)$ and $C_{i}^{\text {ref }}(k)$ of the reference models $\left(\eta_{0}\right.$ and $\eta_{i}$ ) are calculated by the HFEM, and the $C^{\text {ref }}(k)$ of the inspected structure is obtained from ultrasonic experiments. $B_{0}(k)$ and $B_{i}(k)$ denote the integrands of initial reference model and the $i^{\text {th }}$ reference model, respectively. When the difference $\Delta \varepsilon$ between $\eta_{i+1}\left(x_{1}\right)$ and $\eta_{i}\left(x_{1}\right)$ is less than or equal to $M$, the final result $\eta_{i+1}\left(x_{1}\right)$ is achieved. Otherwise, $\eta_{i+1}\left(x_{1}\right)$ in the $(i+1)^{\text {th }}$ iteration will replace $\eta_{i}\left(x_{1}\right)$, and the loop continues. The red lines represent the flow path of the initial reconstruction, and the blue lines express the flow path of the iterations. 


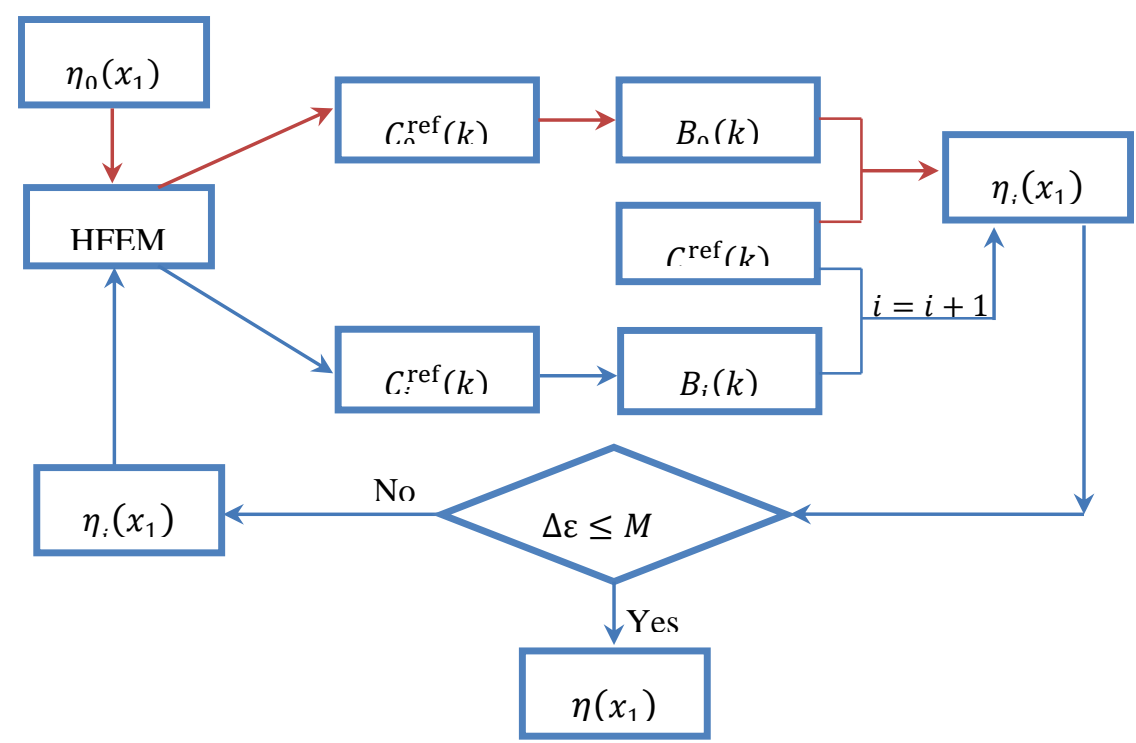

Fig. 2. The flowchart of QDFTU. $\eta_{0}$ and $\eta_{i}$ represent the initial reference model and the $i^{\text {th }}$ reconstructed result, respectively, where $i=1,2,3, \cdots$.

In the process of large-depth defect reconstruction, it is emphasized that the formulation of the convergence criterion $\Delta \varepsilon \leq M$ is critically important to the accuracy and efficiency of the reconstruction, because the convergence criterion directly determines the number of iterations and the validity of convergence. In this paper, the formulation of the convergence criterion is defined as follows,

$\Delta \varepsilon=\sqrt{\frac{\sum_{m=1}^{N}\left[\left(\eta_{i+1}\left(x_{1}^{(m)}\right)-\eta_{i}\left(x_{1}^{(m)}\right)\right)^{2}\right]}{N}}(i=1,2,3, \cdots)$

where $N$ denotes the number of sampling points at the defect boundary, the subscript $i$ indicates the number of iterations, $\Delta \varepsilon$ means the root mean square error (RMSE), to which $M$ is assigned as a threshold value. $\eta_{i+1}\left(\alpha_{1}\right), \eta_{i}\left(\alpha_{1}\right)$, and $\eta_{0}\left(\alpha_{1}\right)$ denote the current, previous and initial reference defect profiles, respectively. If $\Delta \varepsilon \leq M$, the current result will be considered as the final profile. Otherwise, the current defect profile $\eta_{i+1}(x)$ will replace the previous defect profile $\eta_{i}(x)$ until the convergence criterion is satisfied. In this paper, the value of $\Delta \varepsilon$ is suggested to be $0.08 \mathrm{~h}$ (h means the plate thickness). It is certain that the smaller the value $M$ is, the more the iterations are. However, due to some factors, such as the resolution of guided waves, the density of meshing grids, the adopted digital signal processing technique, etc., the threshold value $M$ is typically chosen empirically.

\section{Numerical Experiments}


Two examples have been examined for reconstructions of large-depth defects in plate structures using the developed QDFTU. The defect profiles shown in Fig. 3 are described as: (a) $d=0.20 h, w=1.216 h$; (b) $d=0.733 h, w=1.337 h$, where $\mathrm{h}$ represents the plate thickness. It is noted that the initial reference model with a rectangular defect has been adopted in this paper. The material properties of the plates include density $\rho=7.9320 \times 10^{3} \mathrm{~kg} / \mathrm{m}^{3}$, Lame constants $\lambda=1.1320 \times 10^{11} \mathrm{~Pa}$ and $\mu=8.4302 \times 10^{10} \mathrm{~Pa}$, and the thickness of plates $h=9 \times 10^{-4} \mathrm{~m}$. The first-order mode of SH guided wave (short for SH0) is adopted as the incident wave. The frequency range of incident SH0 is from $1.8005 \times 10^{4} \mathrm{~Hz}$ to $3.9939 \times 10^{6} \mathrm{~Hz}$.

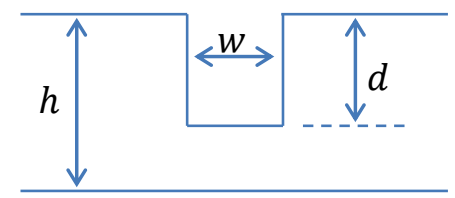

Fig. 3. A plate with a single rectangular defect. $d$ and $w$ indicate the defect depth and width, respectively.
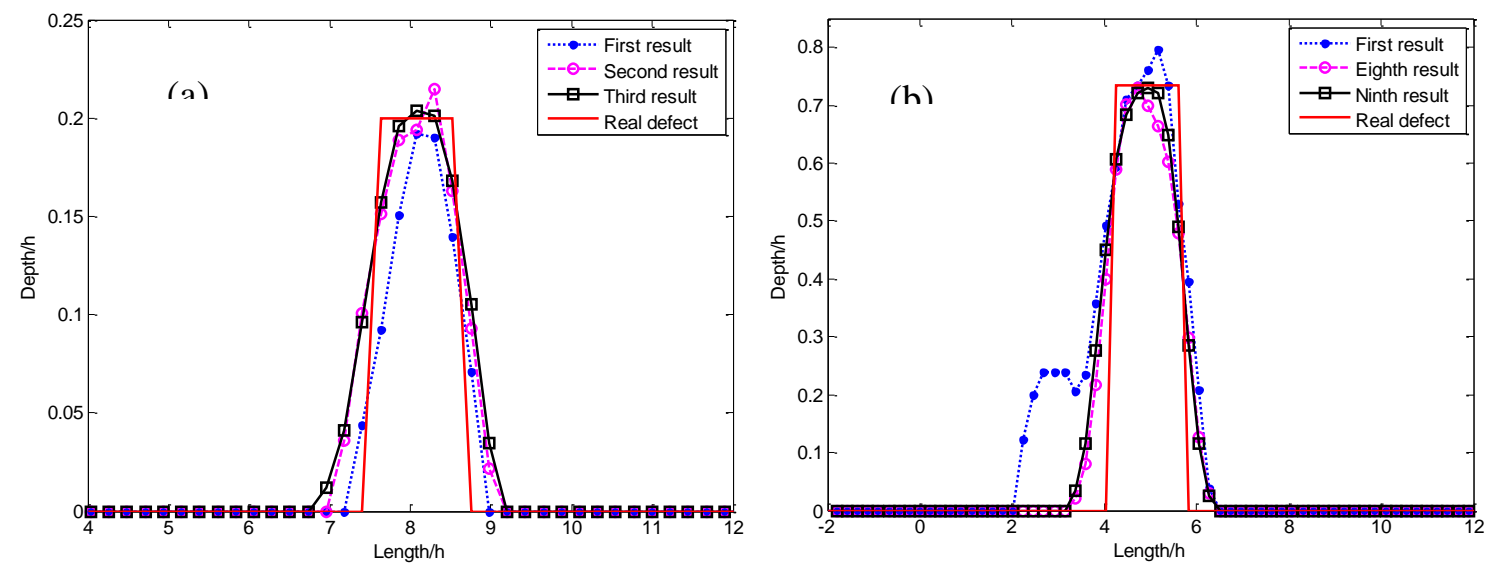

Fig. 4. The reconstruction results of a single rectangular defect by QDFTU. (a) the real shallow defect parameters: $d=0.20 h, w=1.216 h$; (b) the real large-depth defect parameters: $d=0.733 h, w=1.337 h$. In example (a), three numbers of reconstructions have been required to achieve the converged defect profile due to two iterations. For example (b), eight iterations have been executed before the convergence criterion is satisfied. 
To reconstruct a shallow defect shown as Fig. 4(a), a satisfied result can be achieved only within two iterations. When the defect depth $\mathrm{d}$ is increased from $0.20 \mathrm{~h}$ to $0.733 \mathrm{~h}$, the reflection signal gets stronger, which means the current defect should not be viewed as a weak source. Therefore, it can be observed that the first reconstruction result is rather poor, as shown in Fig. 4(b). However, as the number of iterations increases, the final result (the ninth reconstruction) converges to the real defect. By comparison of the results obtained from these two examples, the developed QDFTU method has the ability to efficiently reconstruct defects with a high level of accuracy for strongly scattered structures, i.e. a plate flawed by a large-depth defect. It is concluded that the QDFTU can effectively correct the error introduced by the Born approximation (assumption of weak scattering) for reconstruction of large-depth defects. It is certain that the deeper the defect depth is, the more the required iterations are.

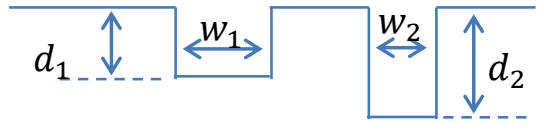

(a)

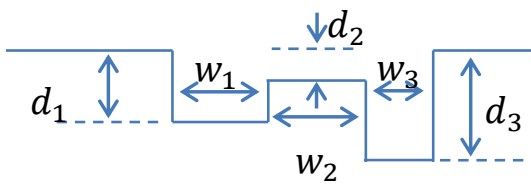

(h)

Fig. 5. The double-rectangular and triple-rectangular defects. (a) $d_{1}=0.30 h, w_{1}=1.333 h, d_{2}=0.50 h$, $w_{2}=1.070 h ;$ (b) $d_{1}=0.333 h, w_{1}=1.333 h, d_{2}=0.20 h, w_{2}=2.367 h, d_{3}=0.533 h, w_{3}=1.366 h$.
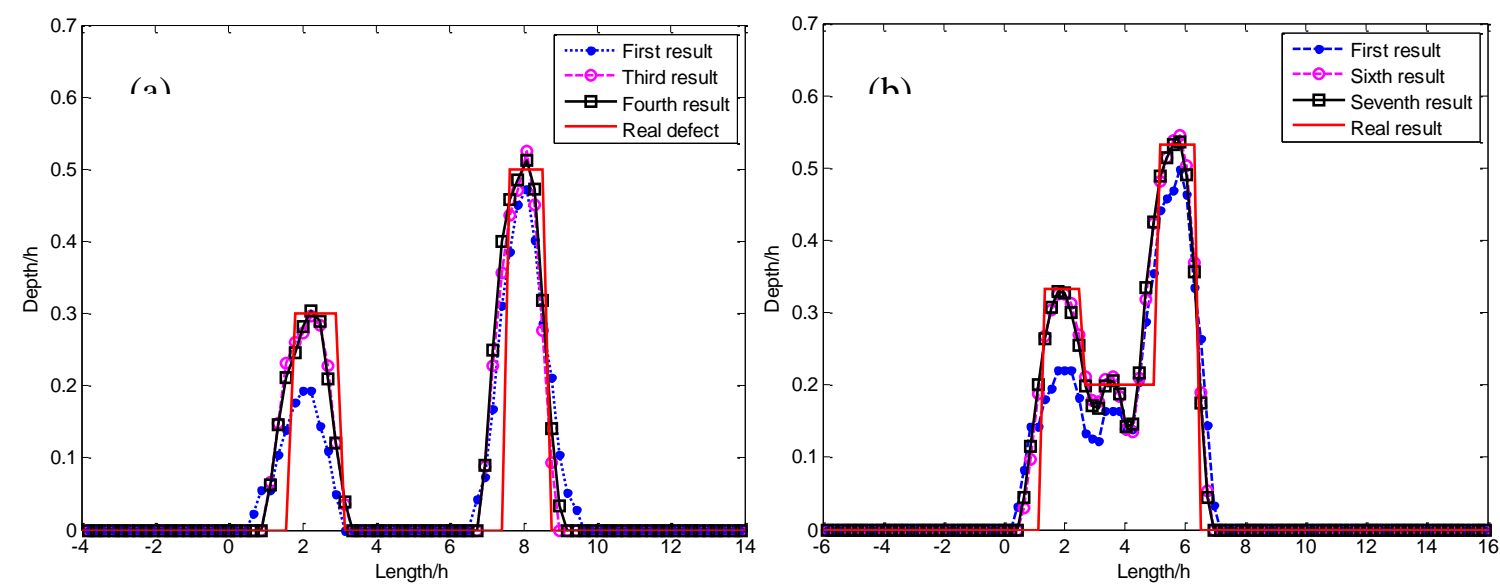

Fig. 6. The reconstruction results of double-rectangular and triple-rectangular defects. (a) the final result is obtained within three iterations; (b) the final result is obtained within six iterations. 
A more challenging problem has been considered in this example. When the number of defects increases and the defect depth reaches more than one half of the plate thickness, the converged result can be obtained within three iterations for reconstruction of a large-depth, double-rectangular defect, as shown in Fig. 6(a). It is noted that the first iteration result regarding the left rectangular defect is obviously less accurate than the real one in Fig. 6(a), because the incident wave propagates from right to left and the monitoring point locates at the right, thus the reflection signal owing to the left defect becomes weak. This phenomenon generally exists in the inspection where the reflection signal of a single direction is received. However, utilizing QDFTU, the left profile can be gradually improved and finally converges to the real one. Similarly, the same conclusion can be observed in Fig. 6(b). For the large-depth, triple-rectangular defect shown in Fig. 6(b), six iterations are executed until the convergence criterion is satisfied. Although the reconstruction result for the middle defect shows a slight discrepancy from the real second defect profile, the entire curve precisely predicts the two main peaks and the defect locations by QDFTU. It is concluded that QDFTU has demonstrated remarkable advantages in reconstructing large-depth defects in plate structures in terms of accuracy and efficiency throughout two challenging examples. For the large-depth defect reconstruction, more iterations are necessary than those required by the shallow defect reconstruction. Obviously, for reconstruction of complex defects, such as the large-depth, triple-rectangular defect, QDFTU needs more iterations to obtain the defect profile with a high level of accuracy, which shows good agreement with the real defect.

\section{Conclusion}

In this paper, a QDFTU method has been proposed to achieve more accurate reconstruction for largedepth defects in plate structures using SH guided waves. To achieve this goal, an updating strategy has been introduced to ensure the accuracy of defect detection. The root mean square error (RMSE) measured by the difference between two consecutive reconstruction profiles is introduced as a convergence criterion for the convergency result. The effectiveness and correctness of the developed QDFTU has been verified by examples of the large-depth flaws and complex defects. It is concluded that the more complex the defect is, the more iterations for reconstruction are required. Also, the defect depth is the most significant factor affecting the number of iterations during the process of reconstruction. It is noted that the limitation of the current work lies in the lack of experimental tests, which will be carried out in the future to further enhance this research work. In summary, QDFTU can compensate for the limitation of the Born approximation and enhance the accuracy of defect profiles, especially for the case that the reflection signal along one single 
direction is received during the inspection. Therefore, the proposed QDFTU has the ability to accurately and efficiently reconstruct large-depth, complex defects in plates using SH guided waves and demonstrates its potential for a wide variety of inspection applications.

Acknowledgements. This work was supported in part by the State Key Laboratory of Mechanics and Control of Mechanical Structures at NUAA [Grant number MCMS-E-0520K02], in part by the Key Laboratory of impact and Safety Engineering, Ministry of Education, Ningbo University [CJ201904], in part by the National Natural Science Foundation of China [Grant numbers 11502108, 1611530686].

\section{References}

[1]Belanger P, Cawley P, Simonetti F. Guided wave diffraction tomography within the born approximation. IEEE Transactions on Ultrasonics, Ferroelectrics, and Frequency Control. 2010; 57(6): 1405-1418.

[2]Wang B, Hirose S. Inverse Problem for Shape Reconstruction of Plate-Thinning by Guided SH-Waves. Materials Transactions. 2012; 53(10):1782-1789.

[3]Yao G, Wu D. Reflection full waveform inversion. Sci. China Earth Sci. 2017; 60: 1783-1794.

[4]Kitahara M, Nakahata K, Hirose S. Elastodynamic inversion for shape reconstruction and type classification of flaws. Wave Motion. 2002; 36(4): 443-455.

[5] van der Neut J, Fokkema JT, van den Berg PM, Zapf M, Ruiter NV, Taskin U, van Dongen KWA. Ultrasonic synthetic-aperture interface imaging. IEEE Transactions on Ultrasonics, Ferroelectrics, and Frequency Control. 2019; 66(5): 888-897.

[6] Tseng H, Lee KH, Becker A. 3D interpretation of electromagnetic data using a modified extended born approximation. Geophysics. 2003; 68(1): 127-137.

[7]Marengo EA, Galagarza ES, Solimene R. Data-driven linearizing approach in inverse scattering. Journal of the Optical Society of America A: Optics and Image Science, and Vision. 2017; 34(9): 1561-1576.

[8]Oliveri G, Salucci M, Anselmi N. Tomographic Imaging of Sparse Low-Contrast Targets in Harsh Environments Through Matrix Completion. IEEE Transactions on Microwave Theory and Techniques. 2018; 66(6): 2714-2730. 
[9]Afsari A, Abbosh AM, Rahmat-Samii Y. Modified Born Iterative Method in Medical Electromagnetic Tomography Using Magnetic Field Fluctuation Contrast Source Operator. IEEE Transactions on Microwave Theory and Techniques. 2019; 67(1): 454-463.

[10]Kaipio J, Huttunen T, Luostari T, Lahivaara T, Monk P. A bayesian approach to improving the born approximation for inverse scattering with high-contrast materials. Inverse Problems. 2019; 35(8): 84001.

[11]Xu S, Shihab Uddin KM, Zhu Q. Improving DOT reconstruction with a born iterative method and USguided sparse regularization. Biomedical Optics Express. 2019; 10(5): 2528-2541.

[12]Sorsa L, Takala M, Eyraud C, Pursiainen S. A Time-Domain Multigrid Solver With Higher-Order Born Approximation for Full-Wave Radar Tomography of a Complex-Shaped Target. IEEE Transactions on Computational Imaging. 2020; 6: 579-590.

[13] Yu L , Giurgiutiu V. In situ 2-D piezoelectric wafer active sensors arrays for guided wave damage detection. Ultrasonics. 2008; 48(2):117-134.

[14] Yu L, Tian Z, Li X, Zhu R, Huang G. Core-skin debonding detection in honeycomb sandwich structures through guided wave wavefield analysis. Journal of Intelligent Material Systems and Structures. $2019 ; 30: 1306-1317$

[15] Yu Z, Xu C, Du F, Cao S, Gu L. Time-domain Spectral Finite Element Method for Wave Propagation Analysis in Structures with Breathing Cracks. Acta Mechanica Solida Sinica. 2020; doi: 10.1007/s10338020-00170-3.

[16] Chen J, Li Z, Gong K. Nondestructive testing method based on lamb waves for localization and extent of damage. Acta Mechanica Solida Sinica. 2017; 30(1): 65-74.

[17]Da Y, Dong G, Shang Y, Wang B, Liu D, Qian Z. Circumferential defect detection using ultrasonic guided waves: An efficient quantitative technique for pipeline inspection. Engineering Computations. 2020; doi:10.1108/EC-06-2019-0260.

[18]Karunasena WM, Shah AH, Datta SK. Plane-strain-wave scattering by cracks in laminated composite plates. Journal of Engineering Mechanics. 1991; 117(8):1738-1754. 
[19]Rattanawangcharoen N, Zhuang W, Shah AH, Datta SK. Axisymmetric guided waves in jointed laminated cylinders. Journal of Engineering Mechanics. 1997; 123(10):1020-1026.

[20]Stoyko DK, Popplewell N, Shah AH. Detecting and describing a notch in a pipe using singularities. International Journal of Solids and Structures. 2014; 51(15-16): 2729-2743.

[21]Shen Y, Giurgiutiu V. Combined analytical FEM approach for efficient simulation of Lamb wave damage detection. Ultrasonics. 2016; 69:116-128.

[22]Bond CZ, Correia CM, Sauvage JF, Neichel B, Fusco T. Iterative wave-front reconstruction in the Fourier domain. Optics Express. 2017; 25(10): 11452.

[23]Haltmeier M, Kowar R, Nguyen LV. Iterative methods for photoacoustic tomography in attenuating acoustic media. Inverse Problems. 2017; 33(11): 115009.

[24]Ye X, Chen X. Subspace-based distorted-born iterative method for solving inverse scattering problems. IEEE Transactions on Antennas and Propagation. 2017; 65(12): 7224-7232.

[25]Hajebi M, Tavakoli A, Dehmollaian M, Dehkhoda P. An iterative modified diffraction tomography method for reconstruction of a high-contrast buried object. IEEE Transactions on Geoscience and Remote Sensing. 2018; 56(7): 4138-4148.

[26] Bao G, Yin T, Zeng F. Multifrequency iterative methods for the inverse medium scattering problems in elasticity. Siam Journal on Scientific Computing. 2019; 41(4): B721-B745.

[27] Poudel J, Na S, Wang LV, Anastasio MA. Iterative image reconstruction in transcranial photoacoustic tomography based on the elastic wave equation. Physics in medicine and biology. 2020; 65(5): 055009.

[28] Huthwaite P, Simonetti F. High-resolution guided wave tomography. Wave Motion. 2013; 50(5): 979_993.

[29] Yang D, Shang X, Malcolm A, Fehler M, Baek H. Image registration guided wavefield tomography for shear-wave velocity model building. Geophysics. 2015; 80(3): U35-U46.

[30] Rao J, Ratassepp M, Fan Z. Guided wave tomography based on full waveform inversion. IEEE Transactions on Ultrasonics, Ferroelectrics, and Frequency Control. 2016; 63(5): 737-745. 
[31] Da Y, Dong G, Wang B, Liu D, Qian Z. A novel approach to surface defect detection. International Journal of Engineering Science. 2018; 133: 181-195.

[32] Zhuang W, Shah AH. Elastodynamic Green's function for laminated anisotropic circular cylinders. Journal of Applied Mechanics. 1999; 66 (3): 665-674 .

[33] Duan W, Gan T. Investigation of guided wave properties of anisotropic composite laminates using a semi-analytical finite element method. Composites Part B. 2019;173:106898

[34] Schmerr LW. Fundamentals of Ultrasonic Nondestructive Evaluation. New York: Plenum Publishing Corporation, 1998. 